\title{
Análisis de productividad de dos carros portacarga en madereo con torre a tala rasa en plantaciones de Pinus radiata
}

\author{
Productivity analysis of two cable yarding carriages in clearcut of Pinus radiata plantations \\ PATRICIO CAREY B. ${ }^{1}$, DIONISIO SOTO H. ${ }^{2}$ \\ ${ }^{1}$ Universidad Austral de Chile, Instituto de Manejo Forestal, Casilla 567, Valdivia, Chile. \\ E-mail: pcarey@uach.cl \\ 2 Ingeniero Forestal, Forestal Arauco, Arauco, Chile.
}

\begin{abstract}
SUMMARY
This study is a comparison of two yarding carriages productivity, in a cable yarding operation, in clearcut of Radiata pine plantations in Chile. A mechanical carriage Christie and the automatic remote control Howe-line carriage were compared working in a Chapman yarder. The forest company introduced the automatic Howe-line carriage in order to include new technology and improve productivity. The study methods consisted of detailed time studies of the productive elements and delays time of the overall work cycle in both extraction operations. The Howe-line carriage production rate was higher than that of the mechanical carriage Christie, with $15.6 \mathrm{~m}^{3} / \mathrm{h}$ and $73.1 \%$, productivity and $11.7 \mathrm{~m}^{3} / \mathrm{h}$ and $73.2 \%$ productivity respectively. The Howe-line carriage will increase the production rate if operational and mechanical delays decrease.
\end{abstract}

Key words: skyline system, productivity, skyline carriages.

\section{RESUMEN}

En el presente estudio se compara la productividad de dos carros portacarga en un sistema de torre de madereo, en cosecha a tala rasa, en bosques de Pinus radiata D. Don. El carro portacarga mecánico, marca Christie y el carro portacarga Howe-line, automático a control remoto, fueron probados en una torre Chapman durante el estudio. Mediante la prueba del carro automático a control remoto Howe-line, se busca avanzar en el desarrollo tecnológico y aumento de la productividad en este tipo de faenas. La metodología utilizada es un estudio de tiempos mediante cronometraje detallado de los tiempos productivos y tiempos de demoras, en los ciclos de trabajo de ambos carros durante las operaciones de extracción. La productividad alcanzada por el carro automático Howe-line es mayor con $15,6 \mathrm{~m}^{3} / \mathrm{h}$ con un porcentaje de productividad de $73,1 \%$, en comparación con la productividad del carro mecánico Christie de $11,7 \mathrm{~m}^{3} / \mathrm{h}$, con un porcentaje de productividad de $73,2 \%$. El carro automático Howe-line puede alcanzar una productividad potencial mayor, si disminuyen las demoras operacionales y mecánicas.

Palabras clave: cables aéreos, productividad, carros portacarga.

\section{INTRODUCCION}

El sector forestal en Chile cumple un rol importante en la economía del país, especialmente en las actividades en plantaciones forestales, princi- palmente de Pinus radiata D. Don, las que están destinadas a los mercados de exportación. De esta manera ha sido necesario permanentemente incorporar nuevas tecnologías con el propósito de obtener una adecuada utilización de estos recursos. 
La mecanización en las faenas de cosecha ha estado orientada principalmente a equipos de madereo terrestre: skidders, cortadoras hidráulicas (feller) y procesadoras (harvester), operando estos equipos en sectores con pendientes de hasta $35 \%$. En sectores donde las condiciones topográficas presentan pendientes mayores, el sistema de madereo con cable aéreo constituye la alternativa técnica factible de extracción de la madera en forma eficiente $(1,2)$.

Frente a este desarrollo tecnológico las empresas no pueden permanecer indiferentes, por lo tanto, deben evaluar constantemente la posibilidad de adquirir nuevos equipos con tecnología de avanzada que les permita, junto con aumentar la productividad en sus operaciones, poder alcanzar menores índices de accidentes, beneficiando de esta forma no sólo al empresario, sino que también a sus trabajadores al brindarles mejores condiciones de trabajo (3).

En Chile, el año 1991, de acuerdo a la información de las empresas forestales, existían 95 torres de madereo (4), de las cuales las más comunes son: Koller, Urus y Chapman, Eeronheimo y Makinen (5). Actualmente en el país existen alrededor de 200 torres de madereo, las cuales trabajan con una variada gama de carros de arrastre, desde los más simples (mecánicos) hasta los carros automáticos radiocontrolados Kahler (6) La configuración de los sistemas de extracción, con cables aéreos, son diversos, siendo uno de los más usados el sistema de cable aéreo vivo, el cual extrae la madera cuesta arriba presentando una adecuada eficiencia, ya que puede aumentar su deflexión con respecto al relieve del terreno.

La empresa Bosques Arauco el año 1969 inicia los sistemas de madereo con torres, mediante el sistema de arrastre (Highlead), para lo cual utiliza la torre de madereo Madill. En el año 1981, la empresa adquiere las torres Chapman EccoLogger, de procedencia canadiense, las cuales incorporan la tecnología del sistema cable aéreo y los carros portacarga mecánicos marca Christie. Las torres Chapman utilizan estos carros hasta fines de los años noventa y posteriormente utilizan a modo de prueba el carro portacarga automático Howe-Line, de procedencia sudafricana, con frenado automático y accionado a control remoto.

Frente a la adquisición de nuevos equipos de producción, las empresas forestales deben anali- zar los beneficios de la inversión, en relación a la productividad y costos del proceso. De esta manera es necesario evaluar las actividades de producción mediante estudios de tiempos, que permitan determinar la productividad en las operaciones y poder comparar los equipos en funcionamiento.

Los estudios de tiempos en las operaciones con sistemas de cables aéreos se clasifican en: tiempos fijos (terminales), tiempos variables (de viajes), tiempos de demoras y tiempos de cambios de instalación $(7,8,9)$.

Los tiempos fijos se definen como aquellos que son independientes respecto a la distancia de madereo, y estos son: los tiempos de carga y descarga, los tiempos de demoras y los tiempos de cambios de instalación.

En relación al tiempo de carga se deben considerar las siguientes variables que afectan el rendimiento tales como: la pendiente del terreno, el número de estroberos (persona que engancha trozos en el bosque), las condiciones del sotobosque e incluso las condiciones del suelo.

En los tiempos de descarga también se considera la existencia de factores de incidencia; Dykstra (9) señala que este tiempo está determinado por el número de trozas por carga, volumen por troza y volumen por ciclo o viaje. Otros autores señalan también como variables de análisis, en los estudios de tiempos en cables aéreos, el peso de la madera y el número de estrobos (elemento para amarre de carga) que se utilizan $(10,11)$.

La mayoría de los autores coincide en definir los tiempos de viaje vacío y viaje cargado como tiempos variables, considerando básicamente la distancia de madereo como el factor determinante en el rendimiento de esta actividad $(8,9)$. El autor Dykstra (9) en el caso del viaje vacío considera, además, la pendiente de la línea o tendido, como variable que influye en el rendimiento.

En operaciones de cosecha forestal con torres de madereo, la producción mensual puede ser muy variable, dependiendo de las condiciones de trabajo tales como: pendiente del terreno, volumen por árbol y condiciones del sotobosque. Un factor importante en la planificación de las operaciones es el tamaño de la cancha de madereo y el grado de congestión de ésta, debido al número de actividades y cantidad de equipos y personal que trabajan en forma simultánea. Otros factores a considerar son el clima, la capacitación del personal, 
BOSQUE 26(2): 95-106, 2005

Análisis de productividad de dos carros portacarga en madereo

el método de trabajo empleado y el tipo de equipo utilizado.

La producción de una torre puede variar desde los 1.500 hasta $4.000 \mathrm{~m}^{3}$ por mes. Los autores Steinlin (12) y Rubio (13), en un estudio del carro automático Howe-line con una torre Koller-600, determinaron tamaño de carga promedio de 2,66 $\mathrm{m}^{3}$ por ciclo y una producción de $2.800 \mathrm{~m}^{3}$ por mes. Neumann y Fuentealba (14), con el mismo carro automático, obtuvieron rendimientos medios del sistema de $24,6 \mathrm{~m}^{3}$ por hora con producciones mensuales de sobre $4.000 \mathrm{~m}^{3}$.

El objetivo de este estudio es desarrollar una evaluación de las ventajas de utilizar un carro automático a control remoto marca Howe-Line, en una torre Chapman, comparado con un carro mecánico tradicional marca Christie, utilizado en la misma torre, en una faena de cosecha a tala rasa en un bosque de Pinus radiata D. Don. El estudio comparativo se basa en un análisis de tiempos y rendimientos por ciclo, y de los tiempos de demoras de ambos equipos.

\section{MATERIAL Y METODOS}

Descripción del área de estudio: El estudio de productividad se desarrolla en el predio Malal y Los Morros perteneciente a Bosques Arauco S.A., el cual se encuentra a unos 30 kilómetros al noreste de la ciudad de Arauco (Chile). El rodal de cosecha tiene una superficie de 35 hectáreas de bosque de Pinus radiata D. Don, el cual presenta un raleo anterior, pero sin poda de los árboles. La topografía del terreno son lomajes suaves a fuertemente quebrados, con pendientes que varían desde un 5 a un $85 \%$. Las laderas presentan pendientes que alcanzan los $600 \mathrm{~m}$ de largo, y otras de menor longitud de $250 \mathrm{~m}$. En el cuadro 1 se indican las características del rodal de cosecha en estudio.

Descripción de los equipos de extracción: El sistema de cosecha utiliza, para la extracción de la madera, una torre de madereo Chapman EccoLogger, de procedencia canadiense y dos carros portacarga: uno mecánico, marca Christie, y otro automático, marca Howe-line, los cuales son objeto de comparación en este estudio. Para estos efectos, en la recolección de datos de tiempos y rendimientos, se emplea la misma cuadrilla de

\section{CUADRO 1}

Características del rodal de cosecha del predio Malal y Los Morros. Harvesting site characteristics Malal y Los Morros.

\begin{tabular}{|ll|}
\hline Especie & Pino radiata \\
\hline Superficie & 5 hectáreas \\
Intervenciones silvícolas & Raleo comercial \\
Edad & 26 años \\
DAP medio & $27,8 \mathrm{~cm}$ \\
Arboles por hectárea & 993 \\
Volumen por árbol & $0,6525 \mathrm{~m}^{3}$ \\
Volumen por hectárea & $648 \mathrm{~m}^{3}$ \\
\hline
\end{tabular}

personal y sólo se cambia el carro portacarga. Las características técnicas de la torre Chapman EccoLogger se presentan en el cuadro 2.

\section{CUADRO 2}

Características técnicas de la torre de madereo Chapman Ecco-Logger.

Technical characteristics of the Chapman Ecco-Logger yarder.

\begin{tabular}{|ll|}
\hline Modelo & Chapman Ecco-Logger \\
\hline Procedencia & Canadá \\
Peso (máquina/línea) & $6.500 \mathrm{~kg} / 2.900 \mathrm{~kg}$ \\
Potencia & $150 \mathrm{Hp}$ \\
Distancia máxima & \\
de extracción & $600 \mathrm{~m}$ \\
Capacidad de carga torre & $3,5 \mathrm{Ton}$ \\
Cable aéreo & $7 / 8^{\prime \prime}$ \\
Cable tracción & $5 / 8 "$ \\
Altura mástil & $14 \mathrm{~m}$ \\
\hline
\end{tabular}

Fuente: Manual del usuario de la torre Chapman.

Carro Howe-Line. El carro portacarga Howe-Line es automático, con control remoto no es motorizado y contiene un mecanismo de fijación a la línea aérea, un mecanismo trabador de la carga, un mecanismo hidráulico y un mecanismo electrónico para la bajada de los estrobos (figura 1).

Carro Christie. El carro portacarga Christie es un carro mecánico (figura 2). Su desplazamiento por 
BOSQUE 26(2): 95-106, 2005

Análisis de productividad de dos carros portacarga en madereo

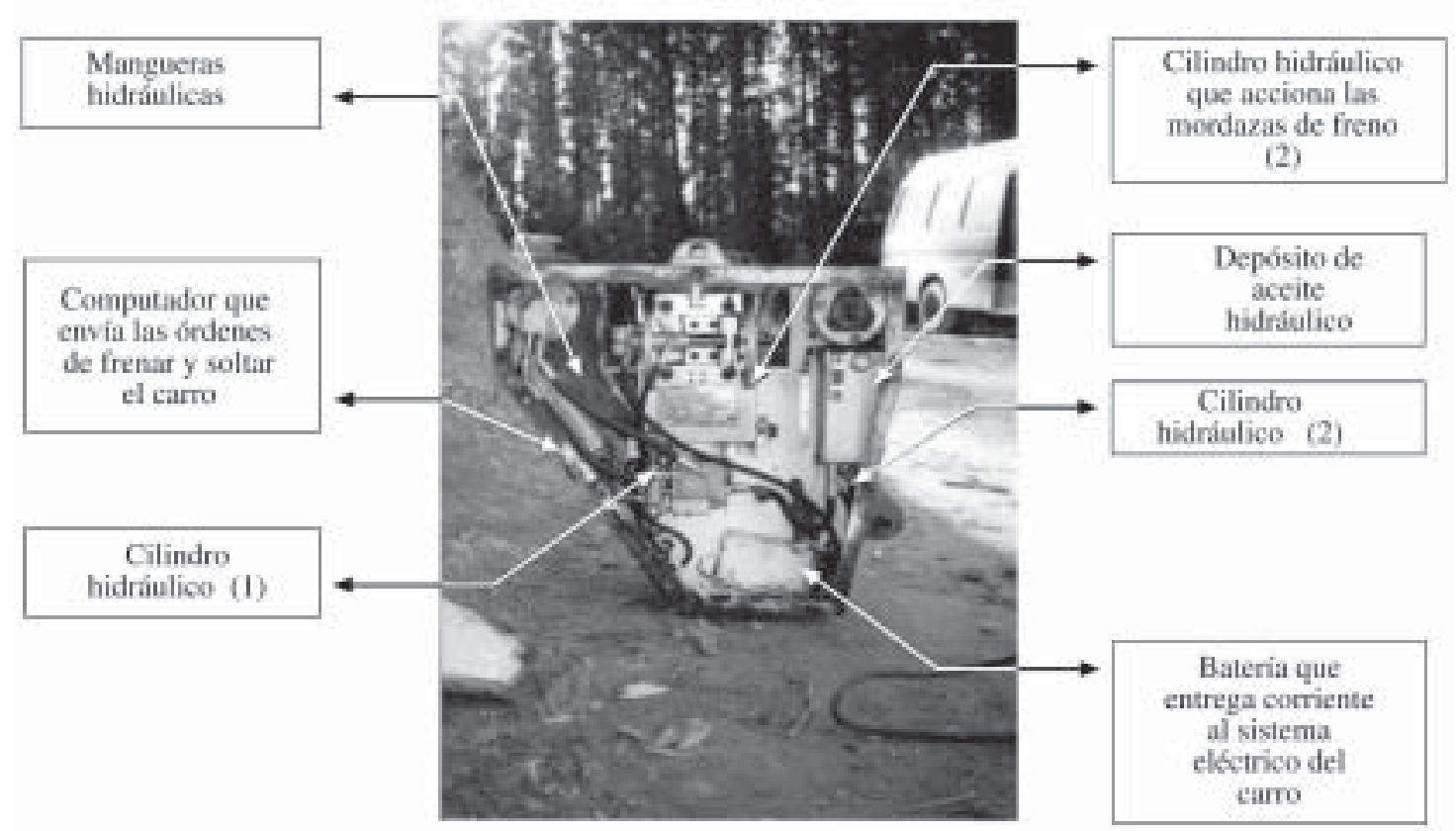

Figura 1. Carro automático Howe-line.

Automatic carriage Howe-line.

el cable aéreo es por gravedad. El mecanismo trabador de la carga se acciona producto de dos sucesos: primero, el carro se detiene por impacto con el tope. El tope es un cuerpo de acero fijado al cable aéreo y permite que el carro portacarga quede fijo al cable aéreo durante el enganche y viaje lateral de la carga de trozas. En la figura 3 se observan los soportes intermedios específicos para cada carro portacarga.
Descripción del sistema de cosecha: El método de corta aplicado corresponde a una tala rasa del bosque de Pinus radiata D. Don. El volteo de los árboles se realiza a base de dos operadores con motosierra, aplicándose un volteo dirigido orientado en el sentido de las curvas de nivel del terreno. Para el enganche de carga en el interior del rodal, se consideran dos estroberos, uno de los cuales tiene la función de jefe de línea. En la

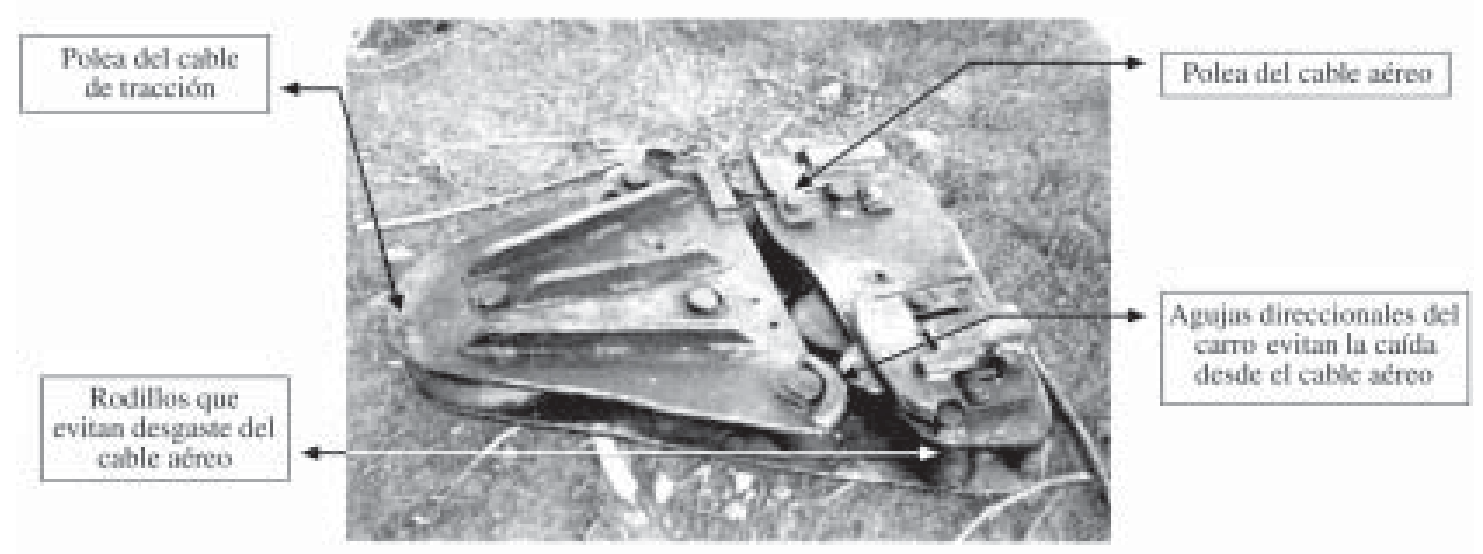

Figura 2. Carro mecánico Christie.

Mechanical carriage Christie. 


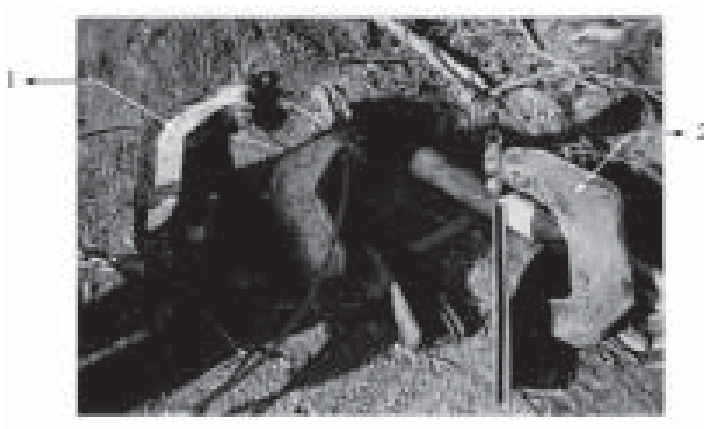

Figura 3. Soportes intermedios: 1. Carro Christie 2. Carro Howe-line.

Intermediate supports: 1 . Christie carriage 2. Howe-line carriage.

cancha de madereo, el sistema considera un operador con motosierra, el cual realiza el trozado de los árboles y un estrobero, que desengancha la carga de la torre. Adicionalmente un cargador frontal con neumáticos en la cancha de madereo clasifica las trozas y carga los camiones.

Estudio de Tiempos: El presente estudio considera en ambos sistemas la medición de los tiempos en la fase de extracción de la madera, con el propósito de comparar la productividad de los carros portacarga, para lo cual se dividió el ciclo de trabajo de la torre de madereo en los siguientes momentos:

1. Viaje vacío: es el tiempo de viaje desde que el carro portacarga inicia el movimiento desde la zona de cancha hasta que se detiene en la zona de amarre; en el caso del carro portacarga mecánico, el término del momento se considera por el impacto del carro con el tope ubicado en el cable aéreo.

2. Toma de los estrobos: es el tiempo que transcurre desde que el carro impacta al tope, liberando y lanzando los estrobos, hasta que éstos son tomados por los estroberos. En el caso del carro mecánico Christie, la toma de los estrobos es totalmente aleatoria, ya que la dirección de caída de los estrobos por el impacto con el tope no es conocida con anterioridad, lo que además implica un mayor desplazamiento de los estroberos en la zona de carga.

3. Amarre de carga: corresponde al tiempo que ocupa el estrobero para enganchar la carga; este momento es igual en ambos sistemas.

4. Huincheo lateral: es el tiempo de viaje lateral de la carga, el que termina cuando el elemento portaestrobo ingresa al carro, liberando las mordazas de freno que lo sujetan al cable aéreo.

5. Viaje cargado: es el tiempo de viaje que el carro portacarga ocupa desde la zona de carga hasta la zona de cancha.

6. Bajada de la carga: es el tiempo que se ocupa en dejar caer la carga desde el carro portacarga a la cancha de madereo.

7. Desamarre de carga: es el tiempo que ocupa el estrobero en desenganchar la carga y termina cuando el cable aéreo es levantado y se inicia un nuevo ciclo.

Las demoras o tiempos no productivos, considerados en el estudio, son los tiempos ocasionales, menores a 10 minutos, que interrumpen el ciclo de trabajo y se clasifican en tres grupos: demoras operacionales, demoras personales y demoras mecánicas. Estas fueron codificadas para facilitar el registro y poder analizar la frecuencia de cada una y su incidencia en la productividad. La clasificación de los tiempos de demoras observados se presentan en el cuadro 3 .

Las demoras operacionales son los tiempos ocasionales que, debido a maniobras o fallas en la organización de la faena, interrumpen la actividad en ejecución, por ejemplo, atascamiento de la carga en un tocón. Las demoras personales son aquellas en que el operador de la torre, los estroberos o los operadores de motosierras, ocupan en pausas o descanso. Finalmente, las demoras mecánicas son aquellas en que por desperfectos mecánicos de los equipos el ciclo de extracción se ve interrumpido, según Carey (11).

\section{RESULTADOS Y DISCUSION}

Las características de las condiciones de trabajo de ambos sistemas se presentan en el cuadro 4.

Se observa una diferencia de los parámetros medios de trabajo, los cuales inciden directamente en la productividad de los sistemas.

Las distancias de trabajo observadas en el carro Christie son menores a las que presenta el tendido del carro Howe-line. Es así como la distancia de extracción observada en el trabajo con el carro Christie es de $136,5 \mathrm{~m}$ y la del carro Howe-line es de 249,6 m. En cuanto a las distan- 
BOSQUE 26(2): 95-106, 2005

Análisis de productividad de dos carros portacarga en madereo

\section{CUADRO 3}

Clasificación de demoras en el ciclo de trabajo de la torre de madereo.

List of delays time in the yarding working cycle.

\begin{tabular}{|lll|}
\hline Operacionales & Mecánicas & Personales \\
\hline Tensado de cable & Problemas carro & No presente el operador \\
Atascamiento en tocón & Aceitar carro & No presente el estrobero \\
Atascamiento en soporte & Corte de cable & $\begin{array}{l}\text { Tiempo de conversación } \\
\text { Mala comunicación }\end{array}$ \\
Cambio soporte & & \\
Pera no ingresó al carro & & \\
Espera por cargador & \\
Espera por motosierra & \\
Tirado de línea & \\
Mal desenganche de carga & \\
Mal enganche de carga & \\
Detención del carro & \\
Se agregó elemento de carga & \\
Objeto a zona de amarre & \\
Fallas del Cacharpete & \\
No caen los estrobos & \\
Se suelta la carga & \\
\hline
\end{tabular}

cias laterales medias observadas estas fueron: para el carro Christie $13,8 \mathrm{~m}$, mientras que la distancia lateral para el carro Howe-line fue de 17,8 m.

\section{CUADRO 4}

Características de las condiciones de trabajo de los carros portacarga.

Characteristics of carriages working conditions.

\begin{tabular}{|lccc|}
\hline & Unidades & Christie & Howe-line \\
\hline Pendiente logitudinal & $\%$ & 44,0 & 40,5 \\
Distancia de madereo & $\mathrm{m}$ & 136,5 & 249,6 \\
Distancia lateral & $\mathrm{m}$ & 13,8 & 17,8 \\
Pendiente lateral & $\%$ & 28,8 & 31,9 \\
Volumen por ciclo & $\mathrm{m}^{3}$ & 1,3 & 2,2 \\
$\mathrm{~N}^{\circ}$ de trozas por ciclo & Unidades & 2,6 & 3,6 \\
\hline
\end{tabular}

La pendiente longitudinal del tendido observada durante el trabajo con el carro Christie es de $44 \%$ y durante el trabajo con el carro Howe-line fue de $40,5 \%$. En cuanto a la pendiente lateral media de trabajo en el tendido del carro Howeline, es de $31,9 \%$ y en el carro Christie es de un $28,8 \%$
En relación a los volúmenes de carga transportados, el carro Christie presenta un volumen medio por ciclo de $1,3 \mathrm{~m}^{3}$ y el carro Howe-line presenta un volumen medio de $2,2 \mathrm{~m}^{3}$ por ciclo. Asociado a lo anterior está el número de trozas transportadas por ciclo, el cual es de 2,6 para el carro Christie y de 3,6 en el carro Howe-line.

Análisis de producción y rendimientos: El estudio considera la medición y registro de 29,09 h de trabajo con el carro mecánico Christie, con 262 ciclos registrados, y 47,79 h de trabajo con el carro automático Howe-line, con 328 ciclos registrados. La información de producción y rendimientos para cada carro portacarga se presenta en el cuadro 5 .

Los rendimientos en ambos sistemas se determinaron a base de la hora productiva y la hora planificada. Por definición, según Carey (11), la hora productiva sólo considera los tiempos de los momentos del ciclo productivo y no se incluyen los tiempos de demoras; de esta manera, el rendimiento en volumen por hora productiva corresponde al rendimiento potencial máximo que puede alcanzar el equipo, sin considerar los tiempos de demoras. Por otra parte, el volumen por hora planificada considera, además de los tiempos pro- 
BOSQUE 26(2): 95-106, 2005

Análisis de productividad de dos carros portacarga en madereo

\section{CUADRO 5}

Producción y rendimientos de los carros portacarga.

Total production and production rates per carriage.

\begin{tabular}{|c|c|c|}
\hline & Christie & Howe-line \\
\hline \multicolumn{3}{|l|}{ Producción } \\
\hline Total de trozas extraídas (unidades) & 682 & 1.196 \\
\hline Volumen total extraído $\left(\mathrm{m}^{3}\right)$ & 348 & 714 \\
\hline Total de tiempo productivo controlado (min) & 1.276 & 2.099 \\
\hline Total de ciclos controlados & 262 & 328 \\
\hline Volumen de carga por ciclo ( $\mathrm{m}^{3} /$ ciclo) & 1,32 & 2,17 \\
\hline Promedio de trozas por ciclo (unidades) & 2,6 & 3,6 \\
\hline Distancia media extracción (m) & 136,5 & 249,6 \\
\hline Distancia máxima extracción (m) & 350 & 500 \\
\hline \multicolumn{3}{|l|}{ Rendimiento } \\
\hline Productividad (\%) & 73,1 & 73,2 \\
\hline Trozas por hora productiva (unidades/hPr) & 32 & 34 \\
\hline Volumen por hora productiva $(\mathrm{m} 3 / \mathrm{hPr})$ & 16,0 & 21,3 \\
\hline Volumen por hora planificada $(\mathrm{m} 3 / \mathrm{hPl})$ & 11,7 & 15,6 \\
\hline
\end{tabular}

ductivos los tiempos de demoras registradas durante el estudio; por lo tanto, corresponde al rendimiento real observado del equipo. El porcentaje de productividad es la relación entre las horas productivas y las horas planificadas registradas en el estudio.

Cabe destacar que la productividad alcanzada por el carro automático Howe-line es de $15,6 \mathrm{~m}^{3} / \mathrm{h}$ con un porcentaje de productividad de $73,1 \%$, en comparación con la productividad del carro mecánico Christie de $11,7 \mathrm{~m}^{3} / \mathrm{h}$, con un porcentaje de productividad de $73,2 \%$.

Es importante destacar que el volumen de carga por ciclo es un $60 \%$ mayor en el carro automático Howe-line, que el del carro mecánico Christie, con $2,17 \mathrm{~m}^{3}$ y $1,32 \mathrm{~m}^{3}$ de carga respectivamente.

Análisis y distribución de los tiempos del ciclo medio: Los tiempos medios por ciclo se presentan en centésimos de minuto en el cuadro 6; se observa que el tiempo de viaje cargado es menor en el carro Christie que en el carro Howe-line, esto se debe principalmente a las diferencias en la distancia de madereo que presentan ambos sistemas, la que es menor para el carro Christie, afectando directamente los tiempos de viaje. La mayor pen- diente existente en el tendido del carro Christie debería tener un efecto negativo sobre el tiempo de viaje cargado, haciéndolo más lento; sin embargo, el menor volumen transportado por este carro hace aparentemente que los viajes sean más rápidos.

\section{CUADRO 6}

Tiempos del ciclo medio de trabajo de los carros portacarga.

Average working cycle times per carriages.

\begin{tabular}{|lrr|}
\hline & Christie* $^{*}$ & Howe-line \\
\hline Viaje vacío & 70,8 & 104,8 \\
Bajada de estrobos & 13,0 & 14,2 \\
Amarre de carga & 190,4 & 190,8 \\
Huincheo lateral & 31,1 & 46,3 \\
Viaje cargado & 114,3 & 184,6 \\
Bajada de carga & 10,1 & 11,3 \\
Subida de línea & 57,7 & 68,1 \\
Tiempo ciclo medio & 487,4 & 620,0 \\
No de casos & 262 & 328 \\
\hline
\end{tabular}

* Unidades en centésimos de minuto. 
En el caso del carro Howe-line, la menor pendiente observada, la mayor distancia de extracción y el mayor volumen de carga por ciclo, implica que los tiempos de viaje cargado son mayores; no obstante, debido al mayor volumen de carga por viaje, permiten que el carro Howeline obtenga un mayor rendimiento $\left(\mathrm{m}^{3} / \mathrm{h}\right)$ en la faena de cosecha analizada.

La distribución de los tiempos del ciclo medio del carro portacarga Christie y del carro Howeline se presentan en la figura 4 .
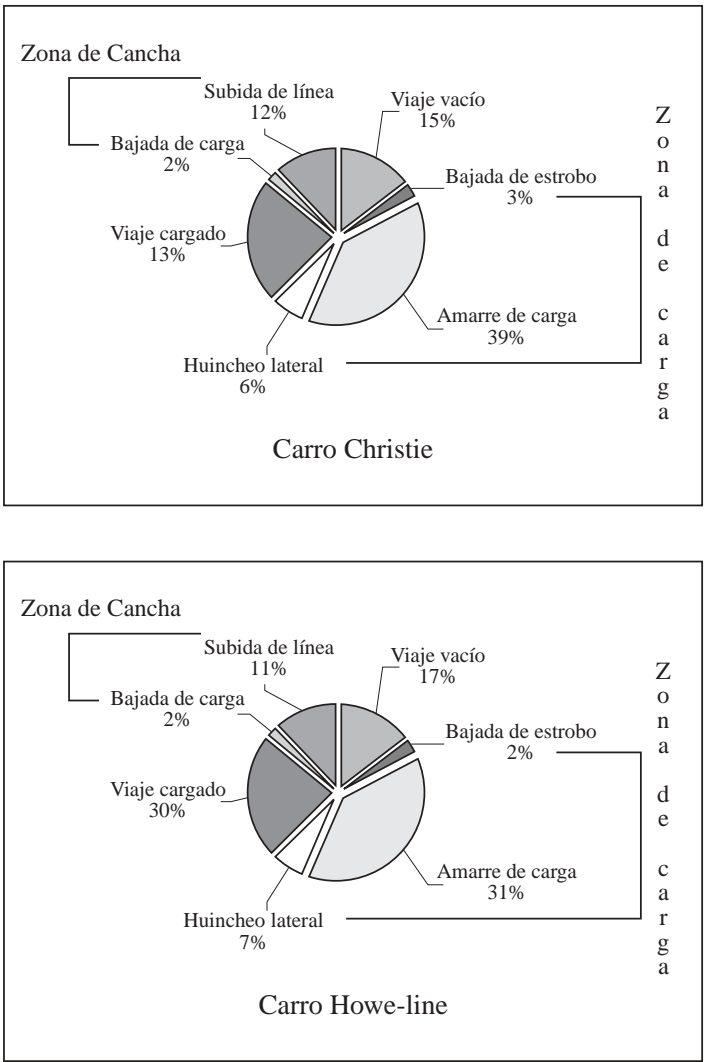

Figura 4. Distribución de los tiempos medios del ciclo de trabajo.

Average working cycle times distribution.

El viaje vacío en el carro Christie representa un $15 \%$ del tiempo del ciclo total con un cálculo de 70,8 centésimos de minuto; en cambio, en el carro Howe-line representa un $17 \%$ con un tiempo de 104,8 centésimos. Este resultado se explica debido a que la distancia media de madereo del carro Howe-line es mayor que la del carro Christie, aumentando los tiempos de viaje del carro automático.
Al analizar el total de los tiempos en la zona de carga, el carro Christie presenta una mayor participación en el tiempo del ciclo total con un $48 \%$, comparado con el carro Howe-line, que alcanza un $40 \%$. No obstante, los tiempos de amarre de carga y tiempos para la bajada de estrobos son mayores en el carro Howe-line, debido a que los estrobos, por control remoto, bajan lentamente desde el carro hacia el estrobero, el cual tiene una ventaja, ya que puede anticipar la ubicación de bajada, lo que permite que el amarre de la carga sea más efectivo, minimizando los tiempos de desplazamiento del estrobero.

Por otra parte, la ventaja que presenta el carro Christie es que, al soltar los estrobos junto con la línea de arrastre, por el impacto del carro con el tope, permite que la línea de arrastre sea expulsada del tambor acumulador de cable, facilitando poder tirar la línea y el desplazamiento del estrobero para amarrar la carga. Por otra parte, el mayor número de trozas por carga y el mayor volumen de carga por ciclo en el carro Howe-line le permiten ser más productivo en esta fase del ciclo.

En la fase de huincheo lateral los tiempos y su participación son: de $6 \%$ y 31,1 centésimos en el carro Christie y $7 \%$ y 46,3 centésimos para el carro Howe-line. No obstante que las distancias medias de huincheo lateral son mayores para el carro Howe-line, este carro es más productivo en esta fase del trabajo, debido a que los volúmenes medios de carga por ciclo extraídos son mayores.

El viaje cargado en el carro Howe-Line tiene un mayor porcentaje de participación en el ciclo con un $30 \%$; esto se debe a la mayor distancia media de madereo y al mayor volumen de carga por ciclo. La bajada de carga es muy similar en ambos carros, lo mismo que el desamarre de carga; en ambos casos los porcentajes de participación son iguales, siendo mayor el tiempo de desamarre de carga en el carro Howe-line, debido al mayor número de trozas a desganchar por viaje.

Análisis de tiempos de demoras: El análisis de los tiempos de demoras de los sistemas por carro portacarga se presenta en los cuadros 7 y 8 . Los tiempos de demoras controlados para el carro Christie fueron de 7,98 h y para el carro Howe-line de 12,79 h. Los tiempos de demoras para el carro Christie se distribuyen en un $83,09 \%$ del tiempo en la categoría de demoras operacionales, un $15,45 \%$ en demoras mecánicas y $1,47 \%$ en demoras personales. 
BOSQUE 26(2): 95-106, 2005 Análisis de productividad de dos carros portacarga en madereo

\section{CUADRO 7}

Estadísticas descriptivas de los tiempos de demoras en el carro Christie.

Descriptives statistics of delay times of the Christie carriage.

\begin{tabular}{|c|c|c|c|c|c|c|c|c|c|}
\hline Clase & Demoras & Suma* & Media & SD & Min & Máx & $\begin{array}{c}\mathrm{N}^{\mathrm{o}} \\
\text { Casos }\end{array}$ & $\begin{array}{c}\% \\
\text { Clase }\end{array}$ & $\begin{array}{c}\% \\
\text { Total }\end{array}$ \\
\hline \multirow[t]{16}{*}{ Operacionales } & Atascamiento tocón & 9.407 & 90,5 & 140,9 & 3 & 604 & 104 & 23,63 & 19,63 \\
\hline & Espera cargador & 8.457 & 151,0 & 169,3 & 10 & 855 & 56 & 21,25 & 17,65 \\
\hline & Mal desestrobado & 6.343 & 83,5 & 53,3 & 11 & 254 & 76 & 15,93 & 13,24 \\
\hline & Espera motosierra & 2.174 & 43,5 & 52,8 & 4 & 335 & 50 & 5,46 & 4,54 \\
\hline & Mala comunicación & 1.873 & 62,4 & 102,0 & 6 & 466 & 30 & 4,70 & 3,91 \\
\hline & Se agregó choco & 1.321 & 101,6 & 144,6 & 25 & 587 & 13 & 3,32 & 2,76 \\
\hline & Objeto a zona amarre & 583 & 291,5 & 206,5 & 85 & 498 & 2 & 1,46 & 1,22 \\
\hline & Falla cacharpete & 313 & 313,0 & 0,0 & 313 & 313 & 1 & 0,79 & 0,65 \\
\hline & Detención en vilo & 171 & 21,3 & 18,8 & 8 & 70 & 8 & 0,43 & 0,36 \\
\hline & Se cortó cable & 40 & 40,0 & 0,0 & 40 & 40 & 1 & 0,10 & 0,08 \\
\hline & Tensado de cable & 2.058 & 343,1 & 312,6 & 20 & 905 & 6 & 5,17 & 4,30 \\
\hline & Atascamiento soporte & 2.221 & 202,0 & 201,2 & 32 & 755 & 11 & 5,58 & 4,64 \\
\hline & Cambio de tope & 1.733 & 433,3 & 70,4 & 345 & 536 & 4 & 4,35 & 3,62 \\
\hline & No cayeron estrobos & 1.312 & 187,4 & 219,4 & 8 & 535 & 7 & 3,30 & 2,74 \\
\hline & La pera no ingresó & 1.181 & 56,2 & 70,5 & 10 & 265 & 21 & 2,97 & 2,46 \\
\hline & Se soltó la carga & 619 & 154,9 & 128,1 & 15 & 353 & 4 & 1,56 & 1,29 \\
\hline Subtotal & & 39.807 & & & & & & & 83,09 \\
\hline \multirow[t]{2}{*}{ Mecánicas } & Se aceitó el carro & 5.879 & 309,4 & 128,5 & 102 & 554 & 19 & 79,43 & 12,27 \\
\hline & Problemas carro & 1.523 & 80,2 & 157,7 & 10 & 707 & 19 & 20,58 & 3,18 \\
\hline Subtotal & & 7.402 & & & & & & & 15,45 \\
\hline \multirow[t]{3}{*}{ Personales } & Conversación & 374 & 124,7 & 63,7 & 42 & 197 & 3 & 52,90 & 0,78 \\
\hline & Estrobero ausente & 303 & 151,5 & 37,5 & 114 & 189 & 2 & 42,86 & 0,63 \\
\hline & Operador ausente & 30 & 15,0 & 5,0 & 10 & 20 & 2 & 4,24 & 0,06 \\
\hline Subtotal & & 707 & & & & & & & 1,47 \\
\hline Total & & 47.916 & & & & & & & 100,00 \\
\hline
\end{tabular}

* Unidades en centésimos de minuto.

Las demoras operacionales en el carro Christie que presentan más frecuencia son: atascamiento de tocón, con un 19,63\% del tiempo total de demoras y una frecuencia de 104 veces; la espera por el cargador frontal en la cancha, con un $17,65 \%$ y una frecuencia de 56 veces, y un mal desenganche o desestrobado, con un $13,24 \%$ y una frecuencia de 76 veces. El atascamiento de tocón se explica debido al arrastre de las trozas hacia arriba contra la pendiente y a la presencia de obstáculos por los residuos de la corta a tala rasa. El tiempo perdido por mal desenganche de carga o desestrobado se debe principalmente a la congestión de trozas en la cancha frente a la torre de madereo. El tiempo de agregar un choco (trozo de madera) al carro es para tener un mayor peso de éste, para el efecto de facilitar el viaje vacío y así obtener una mayor velocidad y no detenerse en lugares de poca pendiente del tendido y, además, facilitar la pasada del carro por los soportes intermedios.

Los tiempos incurridos en enviar objetos a la zona de amarre corresponden a mandar bidón de agua y herramientas varias, los cuales pueden evitarse. El atascamiento en el árbol soporte y el tensado de cable son tiempos de demoras ligadas entre sí, debido a que un atascamiento del carro 
BOSQUE 26(2): 95-106, 2005

Análisis de productividad de dos carros portacarga en madereo

\section{CUADRO 8}

Estadísticas descriptivas de tiempos de demoras en carro Howe-line.

Descriptives statistics of delay times of the Howe-line carriage.

\begin{tabular}{|c|c|c|c|c|c|c|c|c|c|}
\hline Clase & Demoras & Suma* & Media & SD & Min & Máx & $\begin{array}{c}\mathrm{N}^{\mathrm{o}} \\
\text { Casos }\end{array}$ & $\begin{array}{c}\% \\
\text { Clase }\end{array}$ & $\begin{array}{c}\% \\
\text { Total }\end{array}$ \\
\hline \multirow[t]{16}{*}{ Operacionales } & Atascamiento tocón & 28.182 & 117,9 & 148,7 & 5 & 867 & 239 & 38,64 & 36,71 \\
\hline & Tirado de línea & 21.075 & 277,3 & 122,8 & 18 & 627 & 76 & 28,89 & 27,45 \\
\hline & Mal desestrobado & 5.860 & 72,4 & 65,2 & 8 & 437 & 81 & 8,03 & 7,63 \\
\hline & Mala comunicación & 3.357 & 81,9 & 101,0 & 7 & 400 & 41 & 4,60 & 4,37 \\
\hline & Se agregó choco & 2.307 & 72,1 & 45,8 & 18 & 219 & 32 & 3,16 & 3,01 \\
\hline & Espera cargador & 1.440 & 48,0 & 36,1 & 9 & 165 & 30 & 1,97 & 1,88 \\
\hline & Detención en vilo & 1.342 & 44,7 & 72,8 & 8 & 400 & 30 & 1,84 & 1,75 \\
\hline & Falla cacharpete & 1.063 & 354,3 & 58,3 & 272 & 400 & 3 & 1,46 & 1,38 \\
\hline & Objeto a zona amarre & 684 & 228,0 & 63,8 & 152 & 308 & 3 & 0,94 & 0,89 \\
\hline & Espera motosierra & 440 & 31,4 & 28,9 & 7 & 113 & 14 & 0,60 & 0,57 \\
\hline & Mal preestrobado & 211 & 105,5 & 22,5 & 83 & 128 & 2 & 0,29 & 0,27 \\
\hline & Atascamiento soporte & 4.879 & 325,3 & 667,0 & 11 & 2713 & 15 & 6,69 & 6,36 \\
\hline & No caen estrobos & 805 & 35,0 & 31,9 & 7 & 132 & 23 & 1,10 & 1,05 \\
\hline & Tensado de cable & 511 & 73,0 & 77,3 & 17 & 248 & 7 & 0,70 & 0,67 \\
\hline & Cambio soporte & 420 & 420,0 & 0,0 & 420 & 420 & 1 & 0,58 & 0,55 \\
\hline & Se soltó la carga & 363 & 121,0 & 80,2 & 15 & 209 & 3 & 0,50 & 0,47 \\
\hline Subtotal & & 72.939 & & & & & & & 95,01 \\
\hline \multirow[t]{2}{*}{ Mecánicas } & Problema carro & 2.473 & 494,6 & 267,7 & 240 & 911 & 5 & 82,13 & 3,22 \\
\hline & Corte cable & 538 & 269,0 & 231,0 & 38 & 500 & 2 & 17,87 & 0,70 \\
\hline Subtotal & & 3.011 & & & & & & & 3,92 \\
\hline \multirow[t]{3}{*}{ Personales } & Operador ausente & 478 & 79,7 & 100,8 & 7 & 300 & 6 & 58,44 & 0,62 \\
\hline & Estrobero ausente & 315 & 63,0 & 31,2 & 18 & 115 & 5 & 38,51 & 0,41 \\
\hline & Conversación & 25 & 25,0 & 0,0 & 25 & 25 & 1 & 3,06 & 0,03 \\
\hline Subtotal & & 818 & & & & & & & 1,06 \\
\hline Total & & 76.768 & & & & & & & 100,00 \\
\hline
\end{tabular}

* Unidades en centésimos de minuto.

en el árbol soporte implica que se debe bajar el cable aéreo, para desenganchar el carro del árbol y posteriormente debe volver a tensar.

Los tiempos de demoras para el carro Howeline se presentan en el cuadro 8 y se distribuyen en un $95,01 \%$ del tiempo en la categoría de demoras operacionales, un $3,92 \%$ en demoras mecánicas y $1,06 \%$ en demoras personales.

Las demoras operacionales en el carro Howeline que presentan más frecuencia son: atascamiento de tocón, con un $36,71 \%$ del tiempo total de demoras y una frecuencia de 239 veces; el jalado de la línea de arrastre, con un $27,45 \%$ y una fre- cuencia de 76 veces; y un mal desenganche de carga, con un $7,63 \%$ y una frecuencia de 81 veces.

En general, las razones de estos tiempos de demoras son las mismas que las mencionadas anteriormente; no obstante, el tiempo incurrido en el jalado de línea de arrastre en este carro se debe principalmente al tener que desplazarse en algunos sectores del tendido contra la pendiente y transportando la línea de arrastre en forma manual para enganchar la carga de trozos.

En ambos sistemas se observa que una alta proporción de los tiempos de demoras correspon- 
den a las de tipo operacional, lo que indica que, mejorando las actividades de planificación y organización de faena, como también aumentando la capacitación del personal, tendría un alto impacto para aumentar la productividad.

Análisis de tiempos de demoras mayores a 10 minutos: Los tiempos de demoras mayores a 10 minutos se identificaron principalmente en demoras operacionales y demoras mecánicas.

El tiempo de mayor incidencia es la demora operacional que corresponde al cambio de línea; en el caso del carro Christie ésta alcanza a 3,0 h y en el carro Howe-line, alcanza a 2,2 horas.

El tiempo que se incurre en la actividad de cambio de tope sólo se produce en el carro Christie, ya que este lapso no existe para el carro Howeline. Se observó que esta actividad fue realizada eficientemente por la cuadrilla de trabajo, lo que permitió que este tiempo no fuera una diferencia significativa. Los tiempos incurridos en el cambio de árbol soporte se observó que fueron iguales para ambos carros.

El carro Howe-line presentó demoras mecánicas mayores a 10 minutos debido a los elementos eléctricos e hidráulicos en su construcción. Mediante un programa de mantención mecánica preventiva, se pueden obtener aumentos de productividad del sistema.

\section{CONCLUSIONES}

- El incorporar nuevas tecnologías en los sistemas de torre de madereo, como son los carros automáticos, permite aumentar la productividad en corta a tala rasa en plantaciones de Pino radiata.

- La productividad alcanzada por el carro automático Howe-line es mayor con $15,6 \mathrm{~m}^{3} / \mathrm{h}$ con un porcentaje de productividad de $73,1 \%$, en comparación con la productividad del carro mecánico Christie de $11,7 \mathrm{~m}^{3} / \mathrm{h}$, con un porcentaje de productividad de $73,2 \%$. El carro automático Howe-line puede alcanzar una productividad mayor, si disminuyen las demoras observadas, tales como las operacionales y mecánicas mayores a 10 minutos.

- Es importante destacar el volumen de carga por ciclo; en el carro automático Howe-line, es un $60 \%$ mayor que el del carro mecánico
Christie, con $2,17 \mathrm{~m}^{3}$ y $1,32 \mathrm{~m}^{3}$ respectivamente. Esta variable es una gran ventaja del carro automático, lo que le permite una mayor producción por jornada.

- El carro automático Howe-line tuvo una menor incidencia de los tiempos en la zona de carga, con un $40 \%$ del tiempo total del ciclo, no obstante que los tiempos de bajada de línea fueron más altos que los del carro mecánico Christie.

- Los principales tiempos de demoras en ambos equipos fueron demoras operacionales, tales como: atascamiento de carga en tocones, espera por cargador en cancha, bajada de línea, mal desenganche de carga y mala comunicación. Esto implica que mejoras en la planificación, organización y capacitación del personal, tendrían un alto impacto en aumentar la productividad.

- El carro Howe-line presentó tiempos de demoras mecánicas mayores a 10 minutos por su construcción con elementos eléctricos e hidráulicos. Mediante un programa de mantención preventiva del equipo, es posible mejorar la productividad.

\section{BIBLIOGRAFIA}

(1) GIROZ, G., R. RODRIGUEZ. Análisis del límite superior del costo de cosecha con torres de madereo. Actas del II taller de Producción Forestal. Fundación Chile. (Chile), 1989. 9 p.

(2) SILVA, J., W. CABEZAS. Mecanización en una faena de cosecha forestal. Actas III taller de Producción Forestal. Fundación Chile. (Chile), 1991. 15 p.

(3) MARQUEZ, J. Disponibilidad de equipos y sus efectos en la gestión. I Taller de Producción Forestal. Fundación Chile. (Chile), 1988. 19 p.

(4) PARRA, H. Productividad del madereo con torre en faena de raleo comercial. Tesis de Grado. Facultad de Ciencias Forestales. Universidad Austral de Chile. (Chile), 1992. $105 \mathrm{p}$

(5) EERONHEIMO, O., P. MAKINEN. Desarrollo de cosecha forestal en las plantaciones de pino radiata en Chile. Instituto de Investigaciones Forestales de Finlandia (Finlandia), 1995, Serie informativa $\mathrm{N}^{\circ} 542.79 \mathrm{p}$.

(6) KAHLER, A., R. LANDEROS. TL-3000 Una alternativa de madereo cuesta abajo (Down hill). Actas V Taller de Producción Forestal. Fundación Chile (Chile), 1995. $10 \mathrm{p}$

(7) BECKER, J. Proyectos estándares en sistemas actuales de cosecha a tala rasa con torre de madereo Koller K-600. Informe Comité Técnico. Forestal Mininco S.A. (Chile), 1989. 690 p.

(8) CARRASCO, S. Análisis del madereo mediante una torre Koller K-300. Tesis de Grado. Escuela de Ciencias Forestales, Universidad de Chile (Chile), 1984. 95 p. 
BOSQUE 26(2): 95-106, 2005

Análisis de productividad de dos carros portacarga en madereo

(9) DYKSTRA, M. Production rates and cost for yarding by cable, ballon and helicopter compared for clearcutting and partial cutting. Research bulletin 22. Forest Research Laboratory School of Forestry, Oregon State University (USA), 1976. $44 \mathrm{p}$

(10) LEDOUX, C., D. BUTTLER. Simulating cable thinning in young-growth stands. Forest Science Society of American Foresters (USA), 1981, vol. $27 \mathrm{~N}^{\circ}$ 4, p. 745-747.

(11) CAREY, P. Estudio de productividad y rendimiento de sistema de explotación con Skidder. Informe de convenio $N^{\circ} 206$ Forestal Valdivia S.A.-Universidad Austral de Chile. Valdivia (Chile), 1992. 52 p.

(12) STEINLIN, H. La explotación y el transporte de trozos en bosques artificiales de países en desarrollo. El transporte de la madera en países de América Latina. Documento FAO (Italia), 1975, p. 57-58.

(13) RUBIO, F. Estudio de tiempo y rendimiento de tala rasa con torre de madereo Koller K-600 con carro Howe-line. Informe Técnico Forestal Mininco (Chile), 1994. 20 p.

(14) NEUMANN, G., E. FUENTEALBA. Estudio de productividad de madereo con carro mecánico versus carro automático en cosecha con torre de madereo. Actas $V$ Taller de Producción Forestal. Fundación Chile (Chile), 1995. $8 \mathrm{p}$

Recibido: 18.07 .03

Aceptado: 15.04.04 